\section{Neutropenieprophylaxe: Pegfilgrastim nach Plan}

\author{
Muss man es mit den zeitlichen Vorgaben von Pegfilgrastim so genau \\ nehmen, wenn man eine chemotherapieinduzierte febrile Neutropenie \\ (FN) verhindern will? In der Praxis sind Ärzte hier mitunter großzügig. \\ Eine retrospektive Analyse zeigt, welche Folgen das haben kann.
}

D ie Empfehlungen sind eigentlich eindeutig: Um die Entwicklung einer FN infolge einer myelosuppressiven Chemotherapie zu verhindern, sind Koloniestimulierende Faktoren wie Pegfilgrastim sehr effizient. Allerdings sollten sie erst einen Tag nach Ende der Chemotherapie gegeben werden, denn ihre starke Wirkung auf die Proliferation könnte sonst das Risiko für eine FN erhöhen, statt es zu senken. Gleichwohl erhalten immer wieder Patienten die Pegfilgrastim-Prophylaxe am letzten Tag der Chemotherapie - auch aus logistischen Gründen. Schließlich kann gerade für schwerkranke Patienten eine erneute Anreise in die Klinik belastend sein.

In einer umfassenden retrospektiven Analyse untersuchten amerikanische
Kollegen, ob die Pegfilgrastim-Prophylaxe am Ende der Chemotherapie eine Option ist. In die Analyse flossen Daten von mehr als 45.000 erwachsenen Patienten ein, die an einem soliden Tumor oder einem Non-Hodgkin-Lymphom (NHL) litten, eine Chemotherapie mit mittlerem oder hohem FN-Risiko und Pegfilgrastim in mindestens einem Chemotherapiezyklus bekommen hatten.

Zusammen wurden die 45.592 Patienten in 179.152 Zyklen mit Pegfilgrastim behandelt. In $12 \%$ der Zyklen erhielten Patienten Pegfilgrastim an dem Tag, an dem sie auch die letzte Gabe einer Chemotherapie bekamen (Tag 1). In den übrigen Zyklen erhielten die Patienten die Pegfilgrastim-Prophylaxe an den Tagen 2-4 nach Ende der Chemotherapie.
37.095 der Prophylaxe-Behandlungen fanden im 1. Chemotherapie-Zyklus statt. Diese wurden gesondert ausgewertet.

Im Vergleich zur Prophylaxe an den Tagen 2-4 war die Gefahr einer FN höher, wenn Patienten Pegfilgrastim an Tag 1 erhielten: Das galt sowohl für den ersten Zyklus (Odds Ratio [OR] 1,6; p < 0,001) als auch für die Auswertung aller Zyklen zusammen (OR 1,5; $\mathrm{p}<0,001)$.

Fazit: Das Risiko für eine FN ist signifikant erhöht, wenn Patienten mit soliden Tumoren oder einem NHL, die eine Chemotherapie mit mittlerem oder hohem myelosuppressivem Risiko erhalten, am letzten Tag dieser Therapie mit Pegfilgrastim behandelt werden. Günstiger ist die Gabe der Prophylaxe innerhalb von $3 \mathrm{Ta}-$ gen nach Chemotherapieende. Ärzte sollten sich an die empfohlene Administration halten.

Christina Berndt

Weycker D et al. Risk of chemotherapy-induced febrile neutropenia in cancer patients receiving pegfilgrastim prophylaxis: does timing of administration matter? Support Care Cancer. 2016;24(5):2309-16

\section{Effektive Strategien für die Emesisprophylaxe}

\author{
Viele Brustkrebspatientinnen mit moderatem bis hohem Risiko erhalten eine \\ Chemotherapie mit Docetaxel und Cyclophosphamid. Allerdings ist diese \\ Therapie moderat emetogen. Daher wurden verschiedene Strategien zur \\ Emesisprophylaxe untersucht.
}

\footnotetext{
n die prospektive Studie wurden 212 chemotherapienaive Brustkrebspatientinnen mit moderatem bis hohem Risiko aufgenommen, die eine Therapie mit Docetaxel und Cyclophosphamid erhielten. Die antiemetische Therapie bestand im ersten Zyklus aus Dexamethason über 3 Tage und einem $5-\mathrm{HT}_{3}$-Rezeptorantagonisten (Ondansetron, Granisetron, Tropisetron) an Tag 1, entsprechend den Leitlinien der Multinational Association of Supportive Care in Cancer (MASCC).

Der primäre Endpunkt war das vollständige Ansprechen (kein Erbrechen und kein Bedarf an Rescue-Medikation innerhalb der ersten 120 Stunden). Patientinnen, die kein vollständiges Anspre-
}

chen erreichten, wurden in eine einarmige Studie aufgenommen, in der die Wirksamkeit der zusätzlichen Gabe von Aprepitant im zweiten Chemotherapiezyklus untersucht wurde. In beiden $\mathrm{Zy}$ klen wurden die Patientinnen-Tagebücher und der FLIE (Functional Living Index-Emesis) ausgewertet.

Analysiert wurden Daten von $185 \mathrm{~Pa}-$ tientinnen. Im ersten Zyklus sprachen $87 \%$ vollständig auf die antiemetische Standardtherapie an. 23 Patientinnen erhielten im 2. Zyklus Aprepitant und $52,2 \%$ von ihnen sprachen vollständig auf die Therapie an. In Zusammenhang mit der Gabe von Aprepitant wurden keine unerwünschten Ereignisse beobachtet.
Wurde kein vollständiges Ansprechen erreicht, hatte dies einen wesentlichen Einfluss auf die Lebensqualität sowohl in Zyklus 1 als auch in Zyklus 2.

Fazit: Mit einer leitliniengerechten Emesisprophylaxe wurde bei den meisten Brustkrebspatientinnen unter einer Therapie mit Docetaxel und Cyclophosphamid eine Kontrolle von chemotherapieinduzierter Übelkeit und Erbrechen (CINV) erreicht.

Aprepitant erwies sich dabei als eine wirksame Prävention nach dem Versagen der Standard-CINV-Prophylaxe und sollte als Rescue-Therapie bei Patientinnen, die mit einer moderat emetogenen Chemotherapie behandelt werden, in Betracht gezogen werden.

Judith Neumaier

Llombart-Cussac A et al. Incidence of chemotherapy-induced nausea and vomiting associated with docetaxel and cyclophosphamide in early breast cancer patients and aprepitant efficacy as salvage therapy. Results from the Spanish Breast Cancer Group/2009-02 study. Eur J Cancer. 2016;58:122-9. 